\title{
Information Routing in Proteins: The Case of a Therapeutic Antibody ${ }^{+}$
}

\author{
Thomas Tarenzi ${ }^{1,2}$, Marta Rigoli ${ }^{1}$ and Raffaello Potestio ${ }^{1}$ \\ 1 Department of Physics, University of Trento, Povo, Italy \\ 2 Trento Institute for Fundamental Physics and Applications (TIFPA), Povo, Italy \\ + Presented at the Entropy 2021: The Scientific Tool of the 21st Century, 5-7 May 2021; Available online: \\ https://sciforum.net/conference/Entropy2021/.
}

Published: 5 May 2021

Internal dynamics is the link between structure and biological function in proteins [1]. It has been shown that low-frequency dynamics is not only essential for a protein to function [2], but also that a correlation exists between a protein's activity and its specific dynamical properties [3]. Propagation of information between two or more distant sites on the protein network allows concerted, large-scale conformational changes to take place, triggering as a consequence biological responses. In this work, we aim at identifying patterns of information routing within the therapeutic antibody pembrolizumab [4], as communication channels that emerge from the underlying topology and drive the observed correlated motions. Specifically, we focus on the mutual information (MI) of the displacements of atomic positions, as computed from atomistic molecular dynamics simulations, both in presence and in absence of the bound antigen. MI is used to build network models of the antibody for each of the conformational clusters emerging from the simulations; these networks are then interpreted in the light of a graph-theoretical approach, to couple chemical detail and large-scale dynamics. Unveiling interresidue communication pathways in may find application not only in biotechnological manipulation for improved therapeutic agents, but also in design of simplified, multi-resolution antibody models that, describing channels of information transfer at an appropriate high-resolution level, facilitate the dynamical investigation at a lower computational cost [5].

\section{References}

1. Berendsen, H.J.; Hayward, S. Collective protein dynamics in relation to function. Curr. Opin. Struct. Biol. 2000, 10, 165-169.

2. Yang, L.Q.; Sang, P.; Tao, Y.; Fu, Y.X.; Zhang, K.Q.; Xie, Y.H.; Liu, S.Q. Protein dynamics and motions in relation to their functions: Several case studies and the underlying mechanisms. J. Biomol. Struct. Dyn. 2014, 32, 372-393.

3. Hensen, U.; Meyer, T.; Haas, J.; Rex, R.; Vriend, G.; Grubmüller, H. Exploring protein dynamics space: the dynasome as the missing link between protein structure and function. PLoS ONE 2012, 7, e33931.

4. Scapin, G.; Yang, X.; Prosise, W.W.; McCoy, M.; Reichert, P.; Johnston, J.M.; Kashi, R.S.; Strickland, C. Structure of full-length human anti-PD1 therapeutic IgG4 antibody pembrolizumab. Nat. Struct. Mol. Biol. 2015, 22, 953-958.

5. Diggins, I.V.; P; Liu, C.; Deserno, M.; Potestio, R. Optimal coarse-grained site selection in elastic network models of biomolecules. J. Chem. Theory Comput. 2018, 15, 648-664.

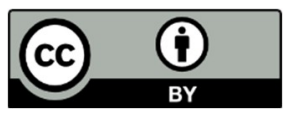

(C) 2021 by the authors. Licensee MDPI, Basel, Switzerland. This article is an open access article distributed under the terms and conditions of the Creative Commons Attribution (CC BY) license (http://creativecommons.org/licenses/by/4.0/). 